\title{
CT Attenuation and Cross-sectional-area Index of the Pectoralis Are Associated With Prognosis in Sarcoma Patients
}

\author{
STEPHANIE $\mathrm{JO}^{1 *}$ and RONNIE SEBRO ${ }^{1,2,3,4^{*}}$ \\ ${ }^{1}$ Department of Radiology, University of Pennsylvania, Philadelphia, PA, U.S.A.; \\ ${ }^{2}$ Department of Orthopaedic Surgery, University of Pennsylvania, Philadelphia, PA, U.S.A.; \\ ${ }^{3}$ Department of Genetics, University of Pennsylvania, Philadelphia, PA, U.S.A.; \\ ${ }^{4}$ Department of Epidemiology, Biostatistics and Informatics, University of Pennsylvania, Philadelphia, PA, U.S.A.
}

\begin{abstract}
Background/Aim: To identify prognostic imaging biomarkers from staging chest computed tomography $(C T)$ in patients with sarcomas. Patients and Methods: CT scans for baseline staging, and surveillance 1-year CT scans in patients newly diagnosed with sarcoma were evaluated. Pectoralis muscle area (PMA), pectoralis muscle index (PMI) and pectoralis CT attenuation density (PMT) were measured. Cox proportional-hazard models were used to determine the association with progression-free survival (PFS) and overall survival (OS). Results: There were 147 patients (53.1\% male) who were followed for a median 1,414 days (range $=219-4851$ days). Approximately $47.6 \%$ (70/147) of patients progressed and $29.9 \%$ (44/147) died. Multivariable Cox-proportional hazards models adjusting for gender, tumor grade and chemotherapy treatment showed that a higher baseline PMT and baseline PMI were associated with increased OS. Conclusion: Higher baseline PMI and PMT are associated with increased overall survival in patients with sarcoma.
\end{abstract}

Sarcomas are rare tumors of mesenchymal origin, and there are approximately 15,000 new cases of bone or soft tissue sarcoma diagnosed annually in the United States (1-3). Sarcomas have a propensity to primarily metastasize to the lungs (3-7). Patients with localized disease have a 5-year life expectancy of approximately $81 \%$, however, patients with metastatic disease have a 5-year life expectancy that is closer to $16 \%$ (3-

This article is freely accessible online.

*Both Authors contributed equally to this work.

Correspondence to: Ronnie Sebro, MD, Ph.D., Department of Radiology, University of Pennsylvania, 3400 Spruce Street, Philadelphia, PA 19104, U.S.A. Tel: +1 2152949512, Fax: +1 2156153316,e-mail: Rsebro@gmail.com

Key Words: Sarcoma, CT attenuation, pectoralis, survival, prognosis, muscle index.
7). Sarcopenia is the loss of muscle mass, quality and strength (8). Patients with cancer often experience muscle loss, which is a key component of cancer cachexia (8). Recent reports have shown that measurements from computed tomography (CT) studies of the abdomen and pelvis can be utilized to evaluate muscle loss (9-15). The L3 skeletal muscle index (SMI) (areal lumbar muscle area at the third lumbar vertebra adjusted for height in meters squared) is commonly used as a proxy for sarcopenia (9-15). Low SMI at L3 has been associated with poor outcomes - decreased overall survival in patients with multiple cancers, including melanoma, breast, uterine, colon and esophageal cancer (9-15).

The accepted treatment for local control of sarcomas is radiation therapy and wide local surgical excision or amputation. Because over $70 \%$ of sarcomas occur in the legs/thigh (1-4), patients with sarcomas often undergo surgery/amputations of the extremities, in particular the lower extremity. These patients experience asymmetric muscle mass loss in the ipsilateral psoas - the predominant hip flexor after amputation/surgical reconstruction of the lower extremity (Figure 1). Because the SMI at L3 includes the psoas, the SMI at L3 is often an inappropriate marker for sarcopenia in most patients with sarcoma.

Patients with other cancers (including melanoma, breast, uterine, colon and esophageal cancer) undergo surveillance CT studies of the abdomen/pelvis as part of routine clinical care (11-15). However, patients with sarcomas usually undergo surveillance chest $\mathrm{CT}$ studies because the lung is the most common site of sarcoma metastasis $(6,7)$. The National Comprehensive Cancer Network (NCCN) guidelines recommend considering $\mathrm{CT}$ studies of the abdomen and pelvis only for surveillance in patients with retroperitoneal liposarcoma, myxoid/round cell liposarcoma, epithelioid sarcoma, angiosarcoma, and leiomyosarcoma. Because of this, most patients with sarcomas do not routinely undergo CT scans of the abdomen/pelvis for surveillance, and therefore, the SMI at L3 cannot be calculated for all sarcoma patients (11-15). 


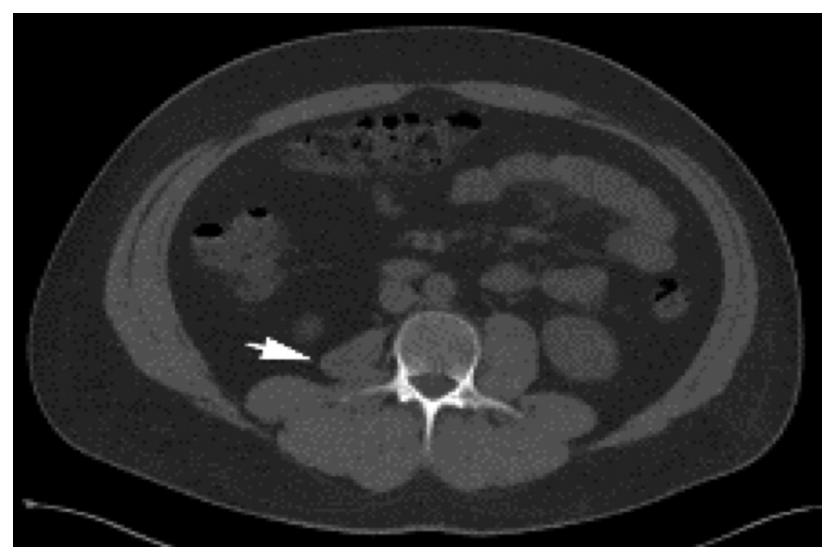

Figure 1. Unenhanced computed tomography $(C T)$ of the abdomen and pelvis demonstrating asymmetric atrophy of the right psoas muscle (white arrow) relative to the left at L3, in a 36-year-old male with an undifferentiated pleomorphic sarcoma of the right thigh after amputation of the right lower extremity 5 years prior. The patient has no evidence of disease.

We hypothesized that imaging biomarkers that are predictive of prognosis in patients with sarcoma, could be obtained opportunistically from these surveillance chest CT studies. The aim of this paper was to assess if there exists a relationship between $\mathrm{CT}$ bone and muscle parameters, and progression-free survival (PFS) and overall survival (OS) in patients with sarcoma.

\section{Patients and Methods}

This retrospective study was Health Insurance Portability and Accountability Act of 1996 (HIPAA) compliant and the study protocol was reviewed and approved by the local institutional review board (IRB). The requirement for signed informed consent from each patient was waived by the IRB.

Patients. This study evaluated patients with newly diagnosed histologically confirmed bone or soft tissue sarcoma, who were treated at a single tertiary academic center between 01/01/2006 and $06 / 01 / 2018$. Patients' age, sex, height and weight at the time of diagnosis were extracted from the medical record. The maximum size of the primary tumor in centimeters at diagnosis, tumor grade, and sarcoma tumor subtype were recorded. Patients were excluded if they had sarcoma involving the T12 vertebral body, thoracic muscles at $\mathrm{T} 12$, or the pectoralis muscles.

Progression-free survival. The length of time in days from the date of histological diagnosis to the development of local recurrence or the development of pulmonary metastases was defined as the progression-free survival (PFS). The time to the development of pulmonary metastases in days was calculated as the time from the date of histological diagnosis (the date the diagnosis was confirmed histologically) until the time pulmonary nodules appeared on the first surveillance chest CT study. If the pulmonary nodules were

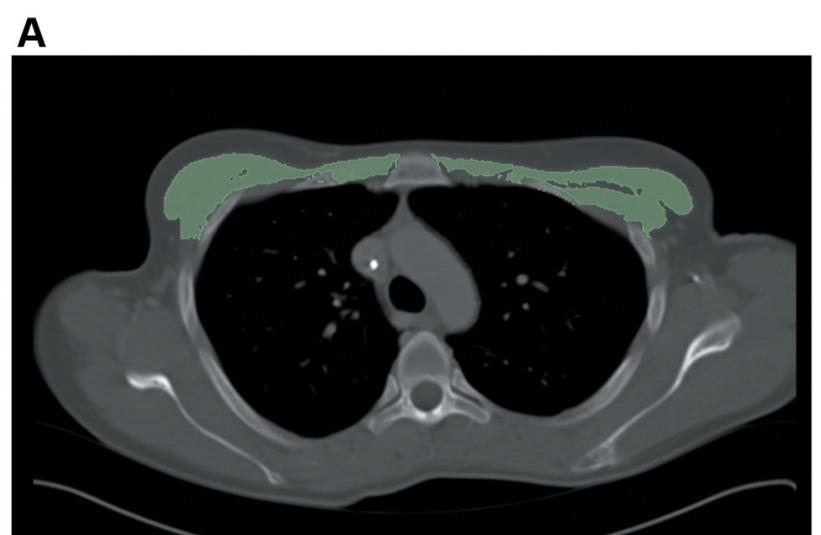

B

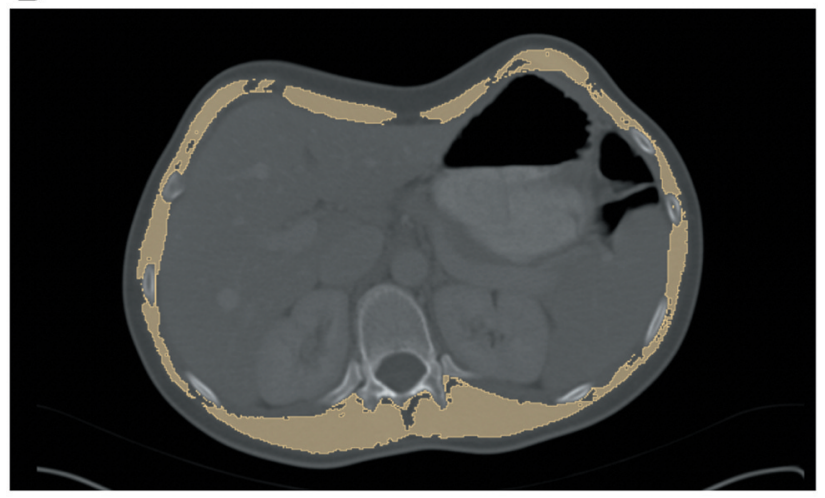

Figure 2. Measurement of muscle cross-sectional area. A) Unenhanced computed tomography $(C T)$ of the chest demonstrating measurement of the pectoralis cross-sectional area (PMA). Green shaded area represents muscle area measured. B) Unenhanced CT of the chest demonstrating measurement of the skeletal muscle cross-sectional area (TMA) at T12. Yellow shaded area represents muscle area measured.

indeterminate on that chest CT study, then the subsequent surveillance chest $\mathrm{CT}$ was reviewed. If the pulmonary nodules were growing in size or if they decreased in size after systemic chemotherapy on the subsequent surveillance chest CT and if the oncologist decided they were clinically consistent with metastases, then the date of the surveillance chest CT where the nodules were first identified was used to define the date of development of pulmonary nodules. If the pulmonary nodules were biopsied and histologically confirmed as metastases, then this date (the date the nodule was first identified on CT) was used as the date of development of pulmonary nodules. The time in days, from diagnosis to histologically confirmed local recurrence was calculated. The minimum time (time to the development of pulmonary metastases compared to the time to local recurrence) was used for the progression-free survival if both conditions occurred. If the patient was lost to follow-up, then the patient was considered censored at the last documented clinic visit/correspondence.

Overall survival. OS was calculated from the date the sarcoma diagnosis was first confirmed histologically until the date the patient was deceased. If the patient was lost to follow-up, then the 
Table I. Demographic and clinical characteristics of study cohort.

\begin{tabular}{|c|c|c|c|c|}
\hline \multirow[b]{2}{*}{ Variable } & \multicolumn{3}{|c|}{ Gender } & \multirow[b]{2}{*}{$p$-Value } \\
\hline & All $(n=147)$ & Male $(\mathrm{n}=78)$ & Female $(n=69)$ & \\
\hline Age in years (SD) & $47.5(16.2)$ & $47.2(17.3)$ & $47.7(14.9)$ & 0.853 \\
\hline Height in m (SD) & $1.72(0.1)$ & $1.75(0.10)$ & $1.67(0.09)$ & $<0.001$ \\
\hline Weight in kg (SD) & $80.7(22.7)$ & $84.1(23.9)$ & $76.8(20.8)$ & 0.052 \\
\hline Location & & & & 0.007 \\
\hline Abdomen/Pelvis & $28(19.0 \%)$ & $9(11.5 \%)$ & $19(27.5 \%)$ & \\
\hline Chest & $13(8.8 \%)$ & $11(14.1 \%)$ & $2(2.9 \%)$ & \\
\hline Head \& Neck & $3(2.0 \%)$ & $3(3.8 \%)$ & $0(0.0 \%)$ & \\
\hline Lower extremity & $79(55.8 \%)$ & $40(51.3 \%)$ & $39(56.5 \%)$ & \\
\hline Retroperitoneal & $2(1.4 \%)$ & $2(2.6 \%)$ & $0(0.0 \%)$ & \\
\hline Upper extremity & $22(15.0 \%)$ & $13(16.7 \%)$ & $9(13.0 \%)$ & \\
\hline Maximum tumor size in cm (SD) & $10.5(6.3)$ & $10.5(6.1)$ & $10.5(6.5)$ & 0.976 \\
\hline Tumor grade, n (\%) & & & & 0.918 \\
\hline Low & $10(6.8 \%)$ & $6(7.7 \%)$ & $4(6.0 \%)$ & \\
\hline Intermediate & $10(6.8 \%)$ & $6(7.7 \%)$ & $4(6.0 \%)$ & \\
\hline High & $115(78.2 \%)$ & $60(76.9 \%)$ & $55(79.7 \%)$ & \\
\hline Ungraded & $12(8.2 \%)$ & $6(7.7 \%)$ & $6(8.7 \%)$ & \\
\hline Chemotherapy, n (\%) & $105(71.4 \%)$ & $58(74.4 \%)$ & $47(68.1 \%)$ & 0.468 \\
\hline Radiation, n (\%) & $75(51.0 \%)$ & $39(50.0 \%)$ & $36(52.2 \%)$ & 0.869 \\
\hline Surgery, n (\%) & $124(84.4 \%)$ & $65(83.3 \%)$ & $59(85.5 \%)$ & 0.821 \\
\hline PMA in $\mathrm{cm}^{2}(\mathrm{SD})$ & $41.68(19.8)$ & $47.5(20.6)$ & $34.8(16.3)$ & $<0.001$ \\
\hline $\mathrm{PMI}$ in $\mathrm{cm}^{2} / \mathrm{m}^{2}$ (SD) & $14.2(6.7)$ & $15.2(6.5)$ & $13.2(6.9)$ & 0.091 \\
\hline PMT in HU (SD) & $40.3(10.1)$ & $41.5(7.9)$ & $38.9(12.1)$ & 0.136 \\
\hline TMA in $\mathrm{cm}^{2}(\mathrm{SD})$ & $85.5(27.2)$ & $95.9(25.3)$ & $73.3(24.2)$ & $<0.001$ \\
\hline TMI in $\mathrm{cm}^{2} / \mathrm{m}^{2}$ (SD) & $28.9(9.0)$ & $30.0(7.8)$ & $27.6(10.1)$ & 0.122 \\
\hline TMT HU (SD) & $37.8(9.0)$ & $38.8(8.5)$ & $36.5(9.4)$ & 0.133 \\
\hline T12 HU (SD) & $201.0(53.6)$ & $202.8(51.6)$ & $198.9(51.5)$ & 0.662 \\
\hline Delta PMA in $\mathrm{cm}^{2}(\mathrm{SD})$ & $-4.32(10.1)$ & $-5.05(10.2)$ & $-3.46(10.0)$ & 0.347 \\
\hline Delta PMI in $\mathrm{cm}^{2} / \mathrm{m}^{2}(\mathrm{SD})$ & $-1.49(3.6)$ & $-1.72(3.3)$ & $-1.23(4.0)$ & 0.443 \\
\hline Delta PMT in HU (SD) & $-1.3(8.8)$ & $-2.29(7.8)$ & $-0.1(9.9)$ & 0.147 \\
\hline Delta TMA in cm2 (SD) & $-3.65(11.7)$ & $-4.94(13.8)$ & $-2.14(8.3)$ & 0.135 \\
\hline Delta TMI in $\mathrm{cm} 2 / \mathrm{m} 2(\mathrm{SD})$ & $-1.26(4.0)$ & $-1.70(4.54)$ & $-0.75(3.2)$ & 0.156 \\
\hline Delta TMT in HU (SD) & $2.4(6.5)$ & $3.14(6.8)$ & $1.57(6.1)$ & 0.148 \\
\hline Delta T12 (HU) & $-18.1(36.8)$ & $-19.2(34.0)$ & $-16.8(40.0)$ & 0.700 \\
\hline
\end{tabular}

Values presented are means (standard deviation) or numbers (percentages \%). HU: Hounsfield Units; y: years; PMA: pectoralis muscle cross-sectional area at the time of diagnosis; PMI: pectoralis muscle index at the time of diagnosis; PMT: pectoralis muscle CT attenuation at the time of diagnosis; TMA: muscle cross-sectional area at T12 at the time of diagnosis; TMI: muscle index at T12 at the time of diagnosis; TMT: mean CT attenuation of the muscles at T12 at the time of diagnosis; T12 HU: T12 vertebral body CT attenuation in HU at the time of diagnosis; Delta PMA: Pectoralis muscle cross-sectional area at 1-year, minus pectoralis muscle cross-sectional area at the time of diagnosis; Delta PMI: Pectoralis muscle index at 1-year, minus pectoralis muscle index at the time of diagnosis; Delta PMT: Pectoralis muscle CT attenuation at 1-year, minus pectoralis muscle CT attenuation at the time of diagnosis; Delta TMA: Muscle cross-sectional area at T12 at 1-year, minus muscle cross-sectional area at T12 at the time of diagnosis; Delta TMI: Muscle index at T12 at 1-year, minus muscle index at T12 at the time of diagnosis; Delta TMT: Mean CT attenuation of the muscles at T12 at 1year minus mean CT attenuation of the muscles at T12 at diagnosis; Delta T12: T12 vertebral body CT attenuation in HU at 1-year minus the T12 vertebral body $\mathrm{CT}$ attenuation in $\mathrm{HU}$ at the time of diagnosis. $p$-Values in bold represent statistically significant values.

patient was considered censored at the last documented clinic visit/correspondence.

Chest CT parameters. Unenhanced chest CTs were performed using a Siemens Somatom AS definition 128 slice CT scanner (Siemens Healthineers, Erlagen, Germany). The following parameters were used: $120 \mathrm{kVp}$, and slice thickness $1.25 \mathrm{~mm}$. The tube current automatically varied depending on body part imaged and patient habitus to limit the radiation dose to the patient. Images were evaluated using the soft tissue kernel. Patients were imaged in the supine position with the arms placed above the head. Scanners were calibrated (tolerance $\pm 4.0 \mathrm{HU}$ ) per the American College of Radiology (ACR) guidelines using phantoms. All scans were obtained in the supine position.

Skeletal muscle measurements. The Digital Imaging and Communications in Medicine (DICOM) files from the CT studies were transferred into a database. CT images were processed using 3D Slicer software, and a previously published semi-automated image analysis method was used to calculate the muscle cross- 
Table II. Correlations between bone and muscle CT measurements at the time of diagnosis.

\begin{tabular}{|c|c|c|c|c|c|c|}
\hline & & \multicolumn{5}{|c|}{ Males } \\
\hline & & TMI & TMT & PMI & PMT & $\mathrm{T} 12 \mathrm{HU}$ \\
\hline \multirow[t]{5}{*}{ Females } & TMI & - & $-0.05(0.694)$ & $0.74(<0.001)$ & $0.15(0.202)$ & $0.01(0.903)$ \\
\hline & TMT & $0.15(0.256)$ & - & $0.07(0.553)$ & $0.83(<0.001)$ & $0.41(<0.001)$ \\
\hline & PMI & $0.86(<0.001)$ & $0.25(0.044)$ & - & $0.27(0.023)$ & $0.23(0.049)$ \\
\hline & PMT & $0.09(0.484)$ & $0.75(<0.001)$ & $0.29(0.023)$ & - & $0.25(0.027))$ \\
\hline & $\mathrm{T} 12 \mathrm{HU}$ & $0.20(0.116)$ & $0.50(<0.001)$ & $0.30(0.017)$ & $0.29(0.017)$ & - \\
\hline
\end{tabular}

Male correlations are in the upper triangle. Female correlations are in the lower triangle. Numbers in parenthesis represent $p$-values. PMI: Pectoralis muscle index at the time of diagnosis; PMT: pectoralis muscle CT attenuation at the time of diagnosis; TMI: muscle index at T12 at the time of diagnosis; TMT: mean CT attenuation of the muscles at T12 at the time of diagnosis; T12 HU: T12 vertebral body CT attenuation in HU at the time of diagnosis. $p$-Values in bold are statistically significant.

sectional areas (16-26). The thoracic skeletal muscle area (TMA) was first calculated. The vertebral body, ribs, outer and inner thoracic fascia were identified at the T12 vertebral level to create an enclosed region of interest. The TMA was the total crosssectional area of pixels within -29 to +150 Hounsfield Units (HU) TMA was then normalized by the patient's height in meters, squared, to obtain the T12 skeletal muscle index (TMI). The mean CT attenuation of the T12 skeletal muscle (TMT) was also calculated (Figure 2A and B). The pectoralis muscle skeletal muscle area (PMA) at the level of the sternomanubrial joint and pectoralis muscle index (PMI) were calculated similarly. The mean CT attenuation of the pectoralis muscles (PMT) were also utilized as predictors in the analysis. The pectoralis muscle was chosen because it is a large muscle that is clearly visible on all chest CT studies. The 1-year change in the TMI (delta TMI), TMT (delta TMT), PMI (delta PMI) and PMT (delta TMT) were calculated by subtracting the initial values from the initial surveillance chest CT study from values from the corresponding 1-year surveillance chest CT study.

Bone measurements. The CT attenuation of a vertebral body is associated with the bone mineral density (BMD) $(27,28)$, and has been associated with poor outcomes in patients with prostate cancer $(27,28)$. A large region of interest (ROI) measuring approximately $1 \mathrm{~cm}$ in diameter was placed over the vertebral body, taking care to avoid the cortex or hemangiomas. The $\mathrm{CT}$ attenuation of $\mathrm{T} 12$ at the initial surveillance CT study (T12 HU) and the 1-year change in the $\mathrm{T} 12 \mathrm{HU}$ were used as predictors in the analysis.

Statistical analyses. Summary statistics for all variables were calculated. Clinical and demographic variables were compared between genders using $t$-tests with unequal variances for quantitative variables, and Fisher's exact tests for qualitative variables. Pearson's correlations were used to assess the correlations between TMI, TMT, PMI, PMT, and T12 HU. Univariate Cox proportional hazards models were used to evaluate the association between the demographic/clinical variables and OS and PFS, analyzing males and females separately. After adjusting for the variables, which were significant in the univariate analysis (grade, chemotherapy), age and gender were used to assess whether the CT muscle and bone measurements remained predictors of PFS and OS in patients with sarcoma. Statistical analysis was performed using R statistical software v3.4 (Vienna, Austria). All statistical tests were two-sided. The Bonferroni correction was utilized to preserve the Type I error rate, and a p-value $<0.005$ was considered statistically significant for the multivariable Cox proportional hazards models.

\section{Results}

There were 147 patients retrospectively identified and followed for an average 1,708 days (range=219-4,851 days). Undifferentiated pleomorphic sarcoma (UPS)/Malignant fibrous histiocytoma (MFH) was the most common subtype $(27 / 147=18.4 \%)$, followed by Ewing sarcoma $(19 / 147=12.9 \%)$, leiomyosarcoma $(18 / 147=12.2 \%)$, synovial sarcoma $\quad(15 / 147=10.2 \%)$ and myxofibrosarcoma $(14 / 147=9.5 \%)$. Approximately $4.1 \% \quad(6 / 147), \quad 16.3 \%$ (24/147), 70/147 (47.6\%), 29.9\% (44/147) and 2.0\% (3/147) of patients were American Joint Committee on Cancer (AJCC) stage I, II, III, IV and unknown, respectively. The mean (standard deviation) age of the patients was 47.5 (16.2) years. Approximately $53.1 \%(78 / 147)$ of the cohort were male. As expected, men were on average taller $(p<0.001)$ than women. All baseline areal muscle measurements were on average larger in men than women. The average PMA $(p<0.001)$ and TMA $(p<0.001)$ were significantly larger in men than women (Table I). Approximately 47.6\% (70/147) of patients progressed and $29.9 \%(44 / 147)$ died of their disease. Older age was negatively associated with lower PMT ( $\mathrm{r}=-$ $0.27, p=0.001)$, lower TMT $(\mathrm{r}=-0.51, p<0.001)$, and lower T12 HU ( $r=-0.60, p<0.001)$.

Among males, PMI was positively correlated with TMI $(\mathrm{r}=0.74, p<0.001)$. PMT and TMT were also correlated $(\mathrm{r}=0.83, p<0.001)$. Among males, T12HU was strongly correlated with PMT $(\mathrm{r}=0.25, p=0.027)$ and TMT $(\mathrm{r}=0.43$, $p<0.001$ ) (Table II). Among females, PMI was also positively correlated with TMI $(\mathrm{r}=0.86, p<0.001)$. T12 HU was also strongly correlated with PMT $(\mathrm{r}=0.29, p=0.017)$ and TMT $(\mathrm{r}=0.50, p<0.001)$. We also found that PMT and TMT were positively correlated within females $(\mathrm{r}=0.75, p<0.001)$. 

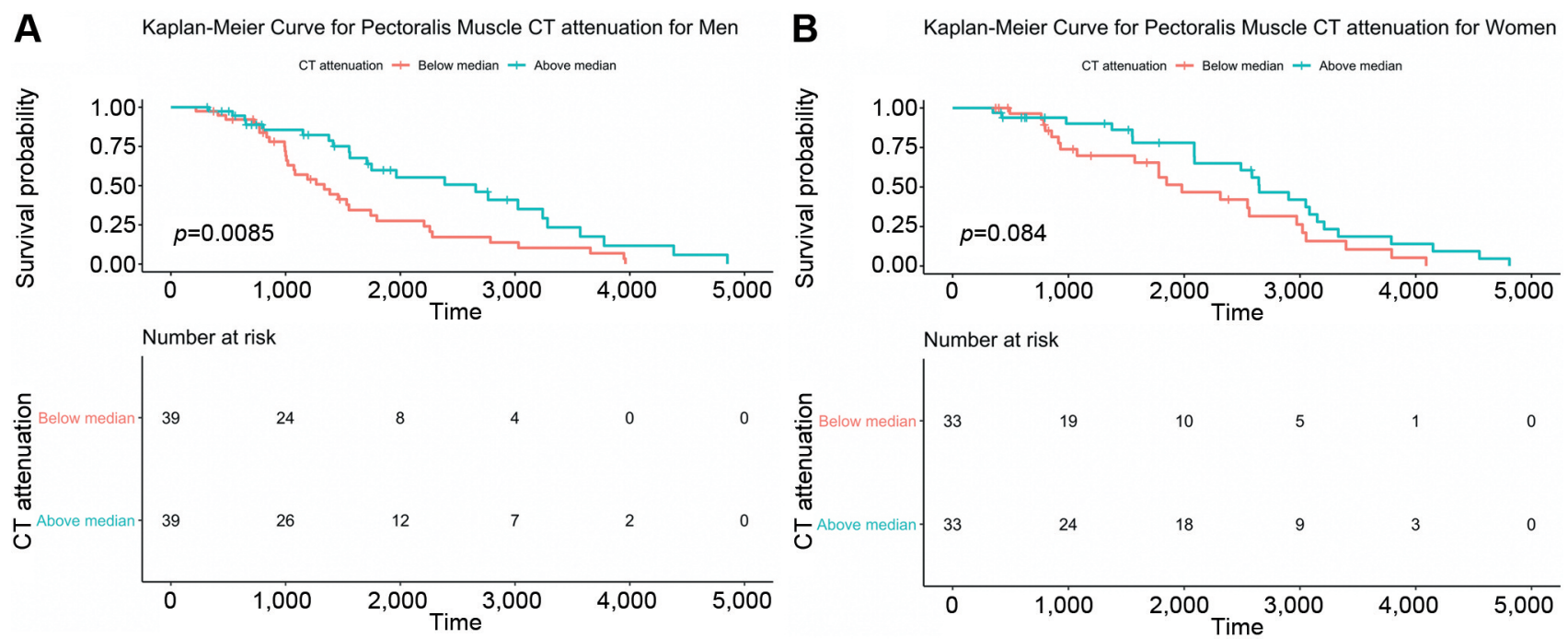

Figure 3. Association between pectoralis muscle computed tomography attenuation and overall survival. A) Kaplan-Meier Curves for sarcoma survival by pectoralis muscle computed tomography attenuation (PMT) for men. p-Value was obtained using the Log-rank test. B) Kaplan-Meier Curves for sarcoma survival by PMT for women. p-Value was obtained using the Log-rank test.
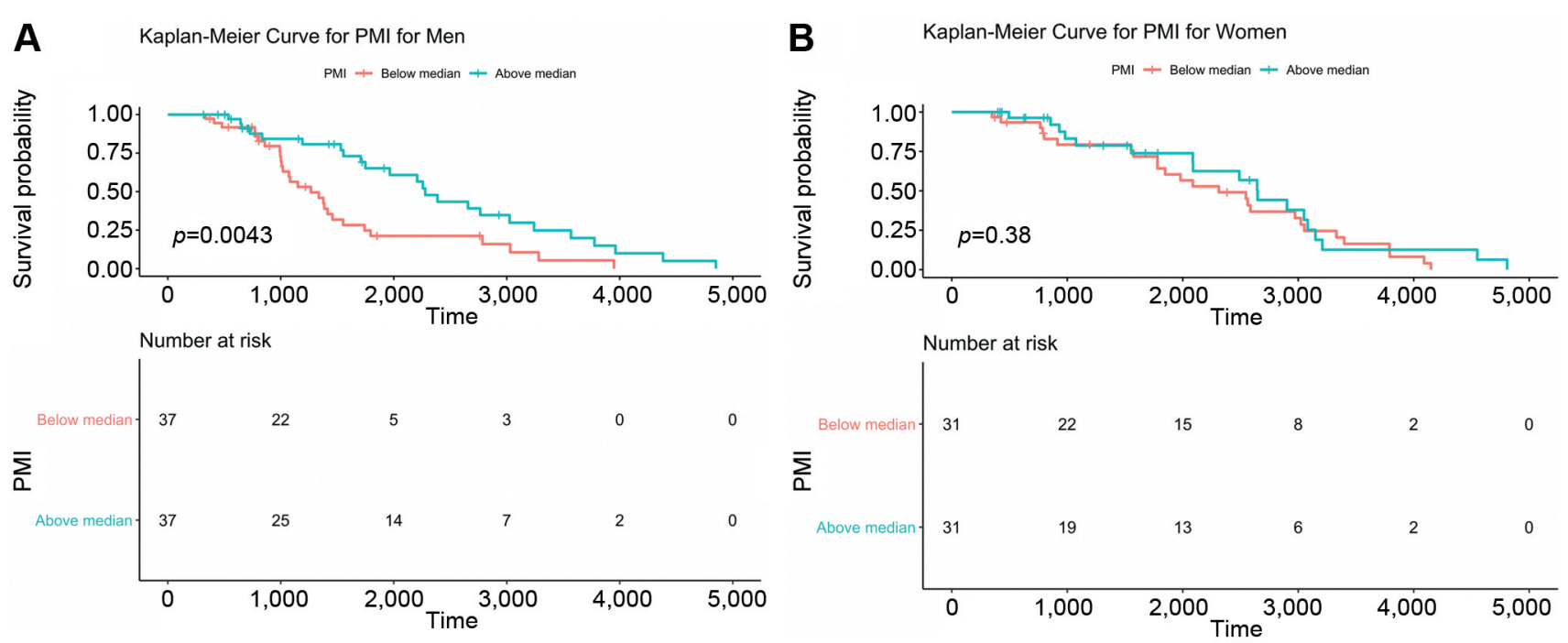

Figure 4. Association between pectoralis muscle index and overall survival. A) Kaplan-Meier Curves for sarcoma survival by pectoralis muscle index (PMI) for men. p-Value was obtained using the Log-rank test. B) Kaplan-Meier Curves for sarcoma survival by PMI for women. p-Value was obtained using the Log-rank test.

Univariate Cox proportional hazards analyses results for PFS and OS are shown in Table III. Kaplan-Meier curves evaluating sarcoma survival are shown for PMT (Figure 3A and B) and PMI (Figure 4A and B). Multivariable Coxproportional hazards models adjusting for age, gender, tumor grade and chemotherapy treatment showed that higher baseline PMT (HR=0.97, 95\% CI=0.95-0.99) and higher baseline PMI $(\mathrm{HR}=0.99,95 \% \mathrm{CI}=0.9987-0.9996)$ were associated with increased OS (Table IV).

\section{Discussion}

The results of this study show that sarcoma patients with higher baseline PMT and PMI had increased OS. This suggests that the amount of baseline cross sectional area and fatty infiltration of the pectoralis musculature were associated with increased OS independent of tumor grade and chemotherapy. The data also show that the CT attenuation of the T12 vertebral body was positively 
Table III. Univariate Cox-proportional hazards analysis predicting progression free survival (PFS) and overall survival (OS) by gender.

\begin{tabular}{|c|c|c|c|c|c|c|c|}
\hline Gender & Variable & PFS Hazard ratio & PFS 95\%CI & PFS $p$-Value & OS Hazard ratio & OS $95 \% \mathrm{CI}$ & OS $p$-Value \\
\hline \multirow[t]{19}{*}{ Males } & Age at diagnosis $(\mathrm{y})$ & 1.01 & $(0.99,1.03)$ & 0.261 & 1.02 & $(1.00,1.03)$ & 0.088 \\
\hline & Height (m) & 5.24 & $(0.15-189.4)$ & 0.365 & 3.75 & $(0.18,78.06)$ & 0.394 \\
\hline & Weight $(\mathrm{kg})$ & 1.00 & $(0.99,1.02)$ & 0.629 & 1.01 & $(1.00,1.02)$ & 0.034 \\
\hline & Maximum tumor size in $\mathrm{cm}$ & 0.99 & $(0.99,1.00)$ & 0.352 & 0.99 & $(0.99,1.00)$ & 0.708 \\
\hline & Tumor grade II & 0.11 & $(0.02,0.67)$ & 0.016 & 0.14 & $(0.04,0.52)$ & 0.003 \\
\hline & Tumor grade III & 0.35 & $(0.11,1.07)$ & 0.066 & 0.20 & $(0.08,0.52)$ & 0.001 \\
\hline & Chemotherapy & 0.72 & $(0.34,1.57)$ & 0.413 & 0.43 & $(0.24,0.78)$ & 0.006 \\
\hline & Radiation & 1.01 & $(0.99,1.97)$ & 0.973 & 1.16 & $(0.68,1.99)$ & 0.579 \\
\hline & Surgery & 0.69 & $(0.26,1.84)$ & 0.455 & 0.76 & $(0.37,1.57)$ & 0.462 \\
\hline & PMI $\left(\mathrm{cm}^{2} / \mathrm{m}^{2}\right)$ & 1.00 & $(1.00,1.00)$ & 0.087 & 0.99 & $(0.99,0.99)$ & 0.004 \\
\hline & PMT (HU) & 0.98 & $(0.94,1.02)$ & 0.415 & 0.97 & $(0.94,1.01)$ & 0.060 \\
\hline & TMI $\left(\mathrm{cm}^{2} / \mathrm{m}^{2}\right)$ & 1.00 & $(1.00,1.00)$ & 0.960 & 0.99 & $(0.99,0.99)$ & 0.420 \\
\hline & TMT (HU) & 0.98 & $(0.94,1.02)$ & 0.421 & 0.97 & $(0.94,1.01)$ & 0.061 \\
\hline & T12 HU (HU) & 0.99 & $(0.99,1.00)$ & 0.050 & 0.99 & $(0.99,1.00)$ & 0.137 \\
\hline & Delta PMI $\left(\mathrm{cm}^{2} / \mathrm{m}^{2}\right)$ & 1.01 & $(1.00,1.01)$ & 0.059 & 1.01 & $(1.00,1.01)$ & 0.002 \\
\hline & Delta PMT (HU) & 0.96 & $(0.91,1.02)$ & 0.157 & 0.97 & $(0.92,1.02)$ & 0.186 \\
\hline & Delta TMI $\left(\mathrm{cm}^{2} / \mathrm{m}^{2}\right)$ & 1.00 & $(1.00,1.01)$ & 0.752 & 1.00 & $(0.99,1.00)$ & 0.228 \\
\hline & Delta TMT (HU) & 1.00 & $(0.99,1.13)$ & 0.091 & 1.04 & $(0.98,1.10)$ & 0.235 \\
\hline & Delta T12 (HU) & 1.00 & $(0.99,1.01)$ & 0.488 & 1.00 & $(1.00,1.01)$ & 0.315 \\
\hline \multirow[t]{19}{*}{ Females } & Age $(y)$ & 1.00 & $(0.98,1.03)$ & 0.665 & 1.01 & $(0.99,1.03)$ & 0.274 \\
\hline & Height (m) & 0.35 & $(0.01,36.02)$ & 0.654 & 1.61 & $(0.03,83.46)$ & 0.812 \\
\hline & Weight $(\mathrm{kg})$ & 1.01 & $(0.99,1.02)$ & 0.507 & 1.01 & $(0.99,1.02)$ & 0.159 \\
\hline & Maximum tumor size in $\mathrm{cm}$ & 0.99 & $(0.99,1.00)$ & 0.841 & 0.99 & $(0.99,1.00)$ & 0.700 \\
\hline & Tumor grade II (Grade I reference) & 0.88 & $(0.08,9.77)$ & 0.917 & 0.70 & $(0.14,3.48)$ & 0.661 \\
\hline & Tumor grade III (Grade I reference) & 0.82 & $(0.11,6.11)$ & 0.844 & 0.45 & $(0.13,1.51)$ & 0.196 \\
\hline & Chemotherapy & 0.61 & $(0.29,1.29)$ & 0.195 & 0.40 & $(0.21,0.77)$ & 0.006 \\
\hline & Radiation & 0.66 & $(0.35,1.25)$ & 0.203 & 0.64 & $(0.36,1.15)$ & 0.133 \\
\hline & Surgery & 0.47 & $(0.20,1.08)$ & 0.073 & 0.87 & $(0.31,2.45)$ & 0.785 \\
\hline & PMI $\left(\mathrm{cm}^{2} / \mathrm{m}^{2}\right)$ & 1.00 & $(1.00,1.01)$ & 0.789 & 0.99 & $(0.99,1.00)$ & 0.534 \\
\hline & PMT (HU) & 0.97 & $(0.94,1.00)$ & 0.029 & 0.97 & $(0.94,0.99)$ & 0.012 \\
\hline & TMI $\left(\mathrm{cm}^{2} / \mathrm{m}^{2}\right)$ & 1.00 & $(1.00,1.01)$ & 0.452 & 1.00 & $(0.99,1.00)$ & 0.388 \\
\hline & TMT (HU) & 0.96 & $(0.92,0.99)$ & 0.026 & 0.96 & $(0.92,0.99)$ & 0.019 \\
\hline & T12 HU (HU) & 0.99 & $(0.99,1.00)$ & 0.076 & 1.00 & $(0.99,1.00)$ & 0.153 \\
\hline & Delta PMI $\left(\mathrm{cm}^{2}\right)$ & 1.00 & $(1.00,1.01)$ & 0.110 & 1.00 & $(1.00,1.01)$ & 0.022 \\
\hline & Delta PMT (HU) & 1.00 & $(0.98,1.03)$ & 0.853 & 1.00 & $(0.98,1.03)$ & 0.616 \\
\hline & Delta TMI $\left(\mathrm{cm}^{2} / \mathrm{m}^{2}\right)$ & 1.00 & $(1.00,1.01)$ & 0.837 & 1.00 & $(0.99,1.00)$ & 0.278 \\
\hline & Delta TMT (HU) & 0.97 & $(0.93,1.02)$ & 0.248 & 0.99 & $(0.94,1.03)$ & 0.607 \\
\hline & Delta T12 (HU) & 1.01 & $(1.00,1.02)$ & 0.037 & 1.01 & $(0.99,1.01)$ & 0.180 \\
\hline
\end{tabular}

HU: Hounsfield Units; y: years; PMA: pectoralis muscle cross-sectional area at the time of diagnosis; PMI: pectoralis muscle index at the time of diagnosis; PMT: pectoralis muscle CT attenuation at the time of diagnosis; TMA: muscle cross-sectional area at T12 at the time of diagnosis; TMI: muscle index at T12 at the time of diagnosis; TMT: mean CT attenuation of the muscles at T12 at the time of diagnosis; T12 HU: T12 vertebral body CT attenuation in HU at the time of diagnosis; Delta PMA: Pectoralis muscle cross-sectional area at 1-year, minus pectoralis muscle crosssectional area at the time of diagnosis; Delta PMI: Pectoralis muscle index at 1-year, minus pectoralis muscle index at the time of diagnosis; Delta PMT: Pectoralis muscle CT attenuation at 1-year, minus pectoralis muscle CT attenuation at the time of diagnosis; Delta TMA: Muscle crosssectional area at T12 at 1-year, minus muscle cross-sectional area at T12 at the time of diagnosis; Delta TMI: Muscle index at T12 at 1-year, minus muscle index at T12 at the time of diagnosis; Delta TMT: Mean CT attenuation of the muscles at T12 at 1-year minus mean CT attenuation of the muscles at T12 at diagnosis; Delta T12: T12 vertebral body CT attenuation in HU at 1-year minus the T12 vertebral body CT attenuation in HU at the time of diagnosis. Bold $p$-values are statistically significant.

correlated with PMI, TMT and PMT in both males and females.

Muscle loss is known to be an independent predictor of survival in patients with hepatocellular carcinoma after hepatectomy (12), stage III melanoma (15), pancreatic adenocarcinoma after resection $(13,14)$, lung adenocarcinoma
(9-11), prostate cancer (29), ovarian cancer (30), breast cancer $(22,31)$, esophageal cancer $(20,21)$, and colorectal cancer (16). Therefore, other studies support our findings, and suggest that surveillance CT studies may have imaging biomarkers that can be used to predict patient prognosis, and show that muscle loss is associated with poor prognosis and decreased overall survival. 
Table IV. Multivariable Cox-proportional hazards analyses predicting progression-free survival (PFS) and overall survival (OS) after adjusting for age, gender, grade and history of prior chemotherapy.

\begin{tabular}{|c|c|c|c|c|c|c|}
\hline Variable & PFS Hazard ratio & PFS 95\%CI & PFS $p$-Value & OS Hazard ratio & OS 95\%CI & OS $p$-Value \\
\hline PMI $\left(\mathrm{cm}^{2} / \mathrm{m}^{2}\right)$ & 0.99 & $(0.99,0.99)$ & 0.004 & 0.99 & $(0.99,0.99)$ & $<0.001$ \\
\hline PMT (HU) & 0.97 & $(0.95,0.99)$ & 0.019 & 0.97 & $(0.95,0.99)$ & 0.005 \\
\hline TMI $\left(\mathrm{cm}^{2}\right)$ & 0.99 & $(0.99,1.00)$ & 0.090 & 1.00 & $(0.99,1.00)$ & 0.179 \\
\hline TMT (HU) & 0.95 & $(0.92,0.99)$ & 0.012 & 0.96 & $(0.94,0.99)$ & 0.016 \\
\hline T12 HU (HU) & 0.99 & $(0.98,0.99)$ & 0.034 & 0.99 & $(0.99,1.00)$ & 0.570 \\
\hline Delta PMI $\left(\mathrm{cm}^{2} / \mathrm{m}^{2}\right)$ & 1.00 & $(0.99,1.00)$ & 0.157 & 1.00 & $(1.00,1.01)$ & 0.014 \\
\hline Delta PMT (HU) & 0.99 & $(0.97,1.01)$ & 0.523 & 0.99 & $(0.97,1.01)$ & 0.421 \\
\hline Delta TMI $\left(\mathrm{cm}^{2} / \mathrm{m}^{2}\right)$ & 1.00 & $(0.99,1.00)$ & 0.984 & 1.00 & $(0.99,1.00)$ & 0.222 \\
\hline Delta TMT (HU) & 0.99 & $(0.95,1.04)$ & 0.736 & 0.97 & $(0.93,1.01)$ & 0.165 \\
\hline Delta T12 (HU) & 1.00 & $(0.99,1.01)$ & 0.056 & 1.00 & $(0.99,1.01)$ & 0.282 \\
\hline
\end{tabular}

HU: Hounsfield Units; y: years; PMA: pectoralis muscle cross-sectional area at the time of diagnosis; PMI: pectoralis muscle index at the time of diagnosis; PMT: pectoralis muscle CT attenuation at the time of diagnosis; TMA: muscle cross-sectional area at T12 at the time of diagnosis; TMI: muscle index at T12 at the time of diagnosis; TMT: mean CT attenuation of the muscles at T12 at the time of diagnosis; T12 HU: T12 vertebral body CT attenuation in HU at the time of diagnosis; Delta PMA: Pectoralis muscle cross-sectional area at 1-year, minus pectoralis muscle crosssectional area at the time of diagnosis; Delta PMI: Pectoralis muscle index at 1-year, minus pectoralis muscle index at the time of diagnosis; Delta PMT: Pectoralis muscle CT attenuation at 1-year, minus pectoralis muscle CT attenuation at the time of diagnosis; Delta TMA: Muscle crosssectional area at T12 at 1-year, minus muscle cross-sectional area at T12 at the time of diagnosis; Delta TMI: Muscle index at T12 at 1-year, minus muscle index at T12 at the time of diagnosis; Delta TMT: Mean CT attenuation of the muscles at T12 at 1-year minus mean CT attenuation of the muscles at T12 at diagnosis; Delta T12: T12 vertebral body CT attenuation in HU at 1-year minus the T12 vertebral body CT attenuation in HU at the time of diagnosis. Bold $p$-values are statistically significant.

One prior study investigating muscle loss in patients with sarcoma found no association between muscle loss assessed by evaluating the height-adjusted psoas CSA at L3 and overall survival (16). However, there are key differences between our study and this prior study. We adjusted for gender, height, and grade rather than just height. We were not interested in estimating sarcopenia - instead we were interested in identifying a CT imaging biomarker that could be used for sarcoma prognosis. Patients with both bone and soft tissue sarcomas were included in our study, whereas in the prior study, only patients with soft tissue sarcoma were evaluated. In addition, we analyzed CT measures of muscle and bone density as continuous variables rather than binary variables - which gives our study more power to detect an association (32).

Muscle loss is a key component of cancer cachexia, and thought to be multifactorial. Environmental causes of muscle loss may include decrease in physical activity and decrease in nutritional intake (33). Patients with progressing sarcoma, like other cancers, may be less active due to fatigue and pain and may suffer from anorexia (33). Muscle loss may be related to decreases in hormones that are important for preservation of muscle mass such as testosterone, dehydroepiandrosterone sulfate (DHEAS), insulin-like growth factor -1 and estrogen (33-38). It is unclear whether decreases in these hormones contribute to muscle loss in patients with sarcoma. Future research is required to assess how all of these factors contribute to muscle loss in patients with sarcoma.
Another interesting finding was that PMI was strongly correlated with the CT measure of bone mineral density in both men and women. One possible explanation for this finding is that individuals that are more active have larger PMI and develop increased BMD, and suggests that bone density and muscle mass are correlated.

This study has a few limitations. This study is based on patients treated at a single tertiary care academic center. The retrospective nature of the study makes it susceptible to ascertainment biases due to referral patterns. Sarcomas are a heterogeneous group of tumors of mesenchymal origin and the optimal treatment depends on the tumor histology. Some tumor types such as osteosarcomas, Ewing sarcoma and myxoid round cell tumors, and metastatic disease are treated with chemotherapy and corticosteroids. Patients with these sarcoma subtypes and metastatic disease may be more susceptible to increased muscle loss because of their treatment, however our analysis adjusted for chemotherapy treatment, which should have mitigated this problem. In conclusion, increased baseline PMI and PMT are associated with better OS after adjusting for patient age, height, tumor grade and chemotherapy in patients with sarcoma.

\section{Conflicts of Interest}

The Authors' declare no financial conflicts of interest in relation to this study. 


\section{Authors' Contributions}

SJ: Data curation, manuscript editing, review and approval of final manuscript. RS: Conceptualization, writing - original draft, manuscript review and editing, formal statistical analysis, methodology, review and approval of final manuscript

\section{Acknowledgements}

RS was supported by R21 NIH/NIMH MH093415.

\section{References}

1 Siegel RL, Miller KD and Jemal A: Cancer statistics, 2019. CA Cancer J Clin 69(1): 7-34, 2019. PMID: 30620402. DOI: $10.3322 /$ caac. 21551

2 Bone and Joint Cancer - Cancer Stat Facts. SEER. Available at: https://seer.cancer.gov/statfacts/html/bones.html [Last accessed on October 1, 2019]

3 Soft Tissue Cancer - Cancer Stat Facts. SEER. Available at: https://seer.cancer.gov/statfacts/html/soft.html [Last accessed on March 27,2019]

4 Cormier JN and Pollock RE: Soft tissue sarcomas. CA Cancer J Clin 54(2): 94-109, 2004. PMID: 15061599. DOI: 10.3322/ canjclin.54.2.94

5 Skubitz KM and D'Adamo DR: Sarcoma. Mayo Clin Proc 82(11): 1409-1432, 2007. PMID: 17976362. DOI: 10.4065/ 82.11.1409

6 Chao C and Goldberg M: Surgical treatment of metastatic pulmonary soft-tissue sarcoma. Oncology (Williston Park) 14(6): 835-841; discussion 842-4, 847, 2000. PMID: 10887634

7 Digesu CS, Wiesel O, Vaporciyan AA and Colson YL: Management of sarcoma metastases to the lung. Surg Oncol Clin N Am 25(4): 721-733, 2016. PMID: 27591495. DOI: 10.1016/ j.soc.2016.05.005

8 Harneshaug M, Benth JS, Kirkhus L, Gronberg BH, Bergh S, Rostoft $\mathrm{S}$ and Slaaen M: CT derived muscle measures, inflammation, and frailty in a cohort of older cancer patients. In Vivo 34(6): 3565-3572, 2020. PMID: 33144469. DOI: 10.21873/ invivo. 12200

9 Kim EY, Kim YS, Park I, Ahn HK, Cho EK, Jeong YM and Kim $\mathrm{JH}$ : Evaluation of sarcopenia in small-cell lung cancer patients by routine chest CT. Support Care Cancer 24(11): 4721-4726, 2016. PMID: 27364150. DOI: 10.1007/s00520-016-3321-0

10 Kim EY, Kim YS, Park I, Ahn HK, Cho EK and Jeong YM: Prognostic Significance of CT-Determined Sarcopenia in Patients with Small-Cell Lung Cancer. J Thorac Oncol 10(12): 1795-1799, 2015. PMID: 26484630. DOI: 10.1097/JTO.000000 0000000690

11 Kinsey CM, San José Estépar R, van der Velden J, Cole BF, Christiani DC and Washko GR: Lower pectoralis muscle area is associated with a worse overall survival in non-small cell lung cancer. Cancer Epidemiol Biomarkers Prev 26(1): 38-43, 2017. PMID: 27197281. DOI: 10.1158/1055-9965.EPI-15-1067

12 Harimoto N, Shirabe K, Yamashita YI, Ikegami T, Yoshizumi T, Soejima Y, Ikeda T, Maehara Y, Nishie A and Yamanaka T: Sarcopenia as a predictor of prognosis in patients following hepatectomy for hepatocellular carcinoma. Br J Surg 100(11): 1523-1530, 2013. PMID: 24037576. DOI: 10.1002/bjs.9258
13 Peng P, Hyder O, Firoozmand A, Kneuertz P, Schulick RD, Huang D, Makary M, Hirose K, Edil B, Choti MA, Herman J, Cameron JL, Wolfgang $\mathrm{CL}$ and Pawlik TM: Impact of sarcopenia on outcomes following resection of pancreatic adenocarcinoma. J Gastrointest Surg 16(8): 1478-1486, 2012. PMID: 22692586. DOI: 10.1007/s11605-012-1923-5

14 Amini N, Spolverato G, Gupta R, Margonis GA, Kim Y, Wagner D, Rezaee N, Weiss MJ, Wolfgang CL, Makary MM, Kamel IR and Pawlik TM: Impact total psoas volume on short- and longterm outcomes in patients undergoing curative resection for pancreatic adenocarcinoma: A new tool to assess sarcopenia. J Gastrointest Surg 19(9): 1593-1602, 2015. PMID: 25925237. DOI: $10.1007 / \mathrm{s} 11605-015-2835-\mathrm{y}$

15 Sabel MS, Lee J, Cai S, Englesbe MJ, Holcombe S and Wang S: Sarcopenia as a prognostic factor among patients with stage III melanoma. Ann Surg Oncol 18(13): 3579-3585, 2011. PMID: 21822551. DOI: 10.1245/s10434-011-1976-9

16 Wilson RJ, Alamanda VK, Hartley KG, Mesko NW, Halpern JL, Schwartz HS and Holt GE: Sarcopenia does not affect survival or outcomes in soft-tissue sarcoma. Sarcoma 2015: 146481, 2015. PMID: 26696772. DOI: 10.1155/2015/146481

17 Nemec U, Heidinger B, Sokas C, Chu L and Eisenberg RL: Diagnosing sarcopenia on thoracic computed tomography: Quantitative assessment of skeletal muscle mass in patients undergoing transcatheter aortic valve replacement. Acad Radiol 24(9): 1154-1161, 2017. PMID: 28365235. DOI: 10.1016/ j.acra.2017.02.008

18 Murray TÉ, Williams D and Lee MJ: Osteoporosis, obesity, and sarcopenia on abdominal CT: a review of epidemiology, diagnostic criteria, and management strategies for the reporting radiologist. Abdom Radiol (NY) 42(9): 2376-2386, 2017. PMID: 28386693. DOI: $10.1007 / \mathrm{s} 00261-017-1124-5$

19 Mourtzakis M, Prado CM, Lieffers JR, Reiman T, McCargar LJ and Baracos VE: A practical and precise approach to quantification of body composition in cancer patients using computed tomography images acquired during routine care. Appl Physiol Nutr Metab 33(5): 997-1006, 2008. PMID: 18923576. DOI: 10.1139/H08-075

20 Prado CM, Lieffers JR, McCargar LJ, Reiman T, Sawyer MB, Martin L and Baracos VE: Prevalence and clinical implications of sarcopenic obesity in patients with solid tumours of the respiratory and gastrointestinal tracts: A population-based study. Lancet Oncol 9(7): 629-635, 2008. PMID: 18539529. DOI: 10.1016/S1470-2045(08)70153-0

21 Boshier PR, Heneghan R, Markar SR, Baracos VE and Low DE: Assessment of body composition and sarcopenia in patients with esophageal cancer: a systematic review and meta-analysis. Dis Esophagus 31(8): 2018. PMID: 29846548. DOI: 10.1093/ dote/doy047

22 Caan BJ, Cespedes Feliciano EM, Prado CM, Alexeeff S, Kroenke $\mathrm{CH}$, Bradshaw P, Quesenberry CP, Weltzien EK, Castillo AL, Olobatuyi TA and Chen WY: Association of muscle and adiposity measured by computed tomography with survival in patients with nonmetastatic breast cancer. JAMA Oncol 4(6): 798-804, 2018. PMID: 29621380. DOI: 10.1001/jamaoncol.2018.0137

23 Caan BJ, Meyerhardt JA, Kroenke CH, Alexeeff S, Xiao J, Weltzien E, Feliciano EC, Castillo AL, Quesenberry CP, Kwan ML and Prado CM: Explaining the obesity paradox: The association between body composition and colorectal cancer survival (C-SCANS Study). Cancer Epidemiol Biomarkers Prev 
26(7): 1008-1015, 2017. PMID: 28506965. DOI: 10.1158/10559965.EPI-17-0200

24 Jacobs AJ, Michels R, Stein J and Levin AS: Improvement in overall survival from extremity soft tissue sarcoma over twenty years. Sarcoma 2015: 279601, 2015. PMID: 25821397. DOI: $10.1155 / 2015 / 279601$

25 Rubbieri G, Mossello E and Di Bari M: Techniques for the diagnosis of sarcopenia. Clin Cases Miner Bone Metab 11(3): 181-184, 2014. PMID: 25568650

26 Sebro R: Obesity, hepatic steatosis, and their impact on fat infiltration of the trunk musculature using unenhanced computed tomography. J Comput Assist Tomogr 41(2): 298-301, 2017. PMID: 28230568. DOI: 10.1097/RCT.0000000000000507

27 Romme EA, Murchison JT, Phang KF, Jansen FH, Rutten EP, Wouters EF, Smeenk FW, Van Beek EJ and Macnee W: Bone attenuation on routine chest CT correlates with bone mineral density on DXA in patients with COPD. J Bone Miner Res 27(11): 2338-2343, 2012. PMID: 22692725. DOI: 10.1002/jbmr.1678

28 Emohare O, Cagan A, Morgan R, Davis R, Asis M, Switzer J and Polly DW Jr: The use of computed tomography attenuation to evaluate osteoporosis following acute fractures of the thoracic and lumbar vertebra. Geriatr Orthop Surg Rehabil 5(2): 50-55, 2014. PMID: 25360331. DOI: 10.1177/2151458514525042

29 McDonald AM, Swain TA, Mayhew DL, Cardan RA, Baker CB, Harris DM, Yang ES and Fiveash JB: CT measures of bone mineral density and muscle mass can be used to predict noncancer death in men with prostate cancer. Radiology 282(2): 475-483, 2017. PMID: 27598538. DOI: 10.1148/radiol.2016160626

30 Gadducci A and Cosio S: The prognostic relevance of computed tomography-assessed skeletal muscle index and skeletal muscle radiation attenuation in patients with gynecological cancer. Anticancer Res 41(1): 9-20, 2021. PMID: 33419795. DOI: 10.21873/anticanres. 14747

31 Villaseñor A, Ballard-Barbash R, Baumgartner K, Baumgartner R, Bernstein L, McTiernan A and Neuhouser ML: Prevalence and prognostic effect of sarcopenia in breast cancer survivors: the HEAL Study. J Cancer Surviv 6(4): 398-406, 2012. PMID: 23054848. DOI: 10.1007/s11764-012-0234-x
32 Altman DG and Royston P: The cost of dichotomising continuous variables. BMJ 332(7549): 1080, 2006. PMID: 16675816. DOI: $10.1136 / \mathrm{bmj} .332 .7549 .1080$

33 Walston JD: Sarcopenia in older adults. Curr Opin Rheumatol 24(6): 623-627, 2012. PMID: 22955023. DOI: 10.1097/BOR.0b01 $3 \mathrm{e} 328358 \mathrm{~d} 59 \mathrm{~b}$

34 McIntire KL and Hoffman AR: The endocrine system and sarcopenia: potential therapeutic benefits. Curr Aging Sci 4(3): 298-305, 2011. PMID: 21529322. DOI: 10.2174/187460981 1104030298

35 Horstman AM, Dillon EL, Urban RJ and Sheffield-Moore M: The role of androgens and estrogens on healthy aging and longevity. J Gerontol A Biol Sci Med Sci 67(11): 1140-1152, 2012. PMID: 22451474. DOI: $10.1093 /$ gerona/gls068

36 Jo E, Lee SR, Park BS and Kim JS: Potential mechanisms underlying the role of chronic inflammation in age-related muscle wasting. Aging Clin Exp Res 24(5): 412-422, 2012. PMID: 22717404. DOI: 10.3275/8464

37 McFarlane C, Plummer E, Thomas M, Hennebry A, Ashby M, Ling N, Smith H, Sharma M and Kambadur R: Myostatin induces cachexia by activating the ubiquitin proteolytic system through an NF-kappaB-independent, FoxO1-dependent mechanism. J Cell Physiol 209(2): 501-514, 2006. PMID: 16883577. DOI: $10.1002 /$ jcp. 20757

38 Baracos VE: Psoas as a sentinel muscle for sarcopenia: a flawed premise. J Cachexia Sarcopenia Muscle 8(4): 527-528, 2017. PMID: 28675689. DOI: $10.1002 /$ jcsm.12221
Received February 2, 2021

Revised February 17, 2021

Accepted February 18, 2021 